\title{
Incompressible surfaces, hyperbolic volume, Heegaard genus and homology
}

\author{
Marc Culler, Jason Deblois and Peter B. Shalen
}

\begin{abstract}
We show that if $M$ is a complete, finite-volume, hyperbolic 3 -manifold having exactly one cusp, and if $\operatorname{dim}_{\mathbb{Z}_{2}} H_{1}\left(M ; \mathbb{Z}_{2}\right) \geq 6$, then $M$ has volume greater than 5.06. We also show that if $M$ is a closed, orientable hyperbolic 3 -manifold with $\operatorname{dim}_{\mathbb{Z}_{2}} H_{1}\left(M ; \mathbb{Z}_{2}\right) \geq$ 4 , and if the image of the cup product map $H^{1}\left(M ; \mathbb{Z}_{2}\right) \otimes$ $H^{1}\left(M ; \mathbb{Z}_{2}\right) \rightarrow H^{2}\left(M ; \mathbb{Z}_{2}\right)$ has dimension at most 1 , then $M$ has volume greater than 3.08. The proofs of these geometric results involve new topological results relating the Heegaard genus of a closed Haken manifold $M$ to the Euler characteristic of the kishkes of the complement of an incompressible surface in $M$.
\end{abstract}

\section{Introduction}

If $S$ is a properly embedded surface in a compact 3-manifold $M$, let $M \backslash \backslash S$ denote the manifold that is obtained by cutting along $S$; it is homeomorphic to the complement in $M$ of an open regular neighborhood of $S$.

The topological theme of this paper is that the bounded manifold obtained by cutting a topologically complex closed simple Haken 3-manifold along a suitably chosen incompressible surface $S \subset M$ will also be topologically complex. Here the "complexity" of $M$ is measured by its Heegaard genus, and the "complexity" of $M \backslash \backslash S$ is measured by the absolute value of the Euler characteristic of its "kishkes" (see Definitions 1.1 below).

Our topological theorems have geometric consequences illustrating a longstanding theme in the study of hyperbolic 3-manifolds — that the volume of a hyperbolic 3-manifold reflects its topological complexity. We obtain lower bounds for volumes of closed and one-cusped hyperbolic manifolds with sufficient topological complexity, extending work of Culler and Shalen along the same lines. Here "topological complexity" is measured in terms of the mod-2 first homology, or the mod-2 cohomology ring. 
Definitions 1.1. We shall say that a 3-manifold $M$ is simple if

(i) $M$ is compact, connected, orientable, irreducible and boundary irreducible;

(ii) no subgroup of $\pi_{1}(M)$ is isomorphic to $\mathbb{Z} \times \mathbb{Z}$; and

(iii) $M$ is not a closed manifold with finite fundamental group.

Let $X$ be a simple 3 -manifold with $\partial X \neq \emptyset$. According to [10] or [11], the characteristic submanifold $\Sigma_{X}$ of $X$ is well defined up to isotopy, and each component of $\Sigma_{X}$ is either an $I$-bundle meeting $\partial X$ in its horizontal boundary (see Definition 3.1), or a solid torus meeting $\partial X$ in a collection of disjoint annuli that are homotopically non-trivial in $X$. We define $\operatorname{kish}(X)$ (the "kishkes" of $X$, or "guts" in the terminology of [2]) to be the union of all components of $\overline{X-\Sigma_{X}}$ that have negative Euler characteristic. The components of $\operatorname{kish}(X) \cap \Sigma_{X}$ are essential annuli in $X$.

If $X$ is a compact 3-manifold whose components are all bounded and simple and if $X_{1}, \ldots, X_{k}$ denote the components of $X$, we define $\operatorname{kish}(X)=$ $\operatorname{kish}\left(X_{1}\right) \cup \cdots \cup \operatorname{kish}\left(X_{k}\right) \subset X$.

Definition 1.2. Let $g$ be an integer $\geq 2$, let $h$ be a positive real number, and let $M$ be an orientable, irreducible 3 -manifold. We shall say that $M$ is $(g, h)$-small if every connected closed incompressible surface in $M$ has genus at least $h$ and every separating connected closed incompressible surface in $M$ has genus at least $g$.

We shall denote the Heegaard genus of a 3 -manifold $Q$ by $\operatorname{Hg}(Q)$.

Theorem 5.1. Suppose $M$ is a closed, simple 3-manifold containing a separating connected closed incompressible surface of some genus $g$, that $\operatorname{Hg}(M) \geq g+4$, and that $M$ is $\left(g, \frac{g}{2}+1\right)$-small. Then $M$ contains a separating connected closed incompressible surface $S$ of genus $g$ satisfying at least one of the following conditions:

(1) at least one component of $M \backslash \backslash S$ is acylindrical; or

(2) for each component $B$ of $M \backslash \backslash S$ we have $\operatorname{kish}(B) \neq \emptyset$.

The key idea in the proof is an organizing principle for cylinders properly embedded in the complement of a separating connected closed incompressible surface. This is discussed in Sections 4 and 5. We apply Theorem 5.1 in conjuction with the theorem below concerning non-separating surfaces, 
which is proved in Section 3. For a manifold $M$ with (possibly empty) boundary, let $\chi(M)$ denote the Euler characteristic of $M$, and let $\bar{\chi}(M)=$ $-\chi(M)$.

Theorem 3.1. Let $M$ be a closed, simple 3-manifold containing a nonseparating connected closed incompressible surface $S$ of genus $g$. Suppose that $\bar{\chi}(\operatorname{kish}(M \backslash \backslash S))<2 g-2$, and that $M$ is $(2 g-1, g)$-small. Then $\operatorname{Hg}(M) \leq$ $2 g+1$.

In a closed, simple 3-manifold, every connected closed incompressible surface has genus at least 2 . Thus any such manifold is $(2,2)$-small. Hence applying Theorems 5.1 and 3.1 to a manifold containing an embedded surface of genus 2, we will easily obtain the following corollary.

Corollary 5.1. Suppose that $M$ is a closed, simple 3-manifold which contains a connected closed incompressible surface of genus 2 , and that $\mathrm{Hg}(M) \geq 6$. Then $M$ contains a connected closed incompressible surface $S$ of genus 2 such that either $M \backslash \backslash S$ has an acylindrical component, or $\bar{\chi}(\operatorname{kish}(M \backslash \backslash S)) \geq 2$.

This corollary will suffice for the geometric applications in this paper. In a future paper, we will apply Theorems 3.1 and 5.1 to the case of a genus 3 surface.

In combination with work of Agol-Storm-Thurston [2] and KojimaMiyamoto [12], Corollary 5.1 implies the following volume bound for sufficiently complex hyperbolic Haken manifolds.

Theorem 6.1. Let $M$ be a closed, orientable hyperbolic 3-manifold containing a closed connected incompressible surface of genus 2, and suppose that $\operatorname{Hg}(M) \geq 6$. Then $M$ has volume greater than 6.45 .

Theorem 6.1 implies Theorems 6.2 and 6.3 below, which extend earlier work of Culler-Shalen.

Theorem 6.2. Let $M$ be a complete, finite-volume, orientable hyperbolic 3-manifold having exactly one cusp, and suppose that

$$
\operatorname{dim}_{\mathbb{Z}_{2}} H_{1}\left(M ; \mathbb{Z}_{2}\right) \geq 6
$$

Then $M$ has volume greater than 5.06 . 
Theorem 6.1 is an improvement on [7, Proposition 10.1]. There, the stronger lower bound of 7 on the dimension of $\mathbb{Z}_{2}$-homology gives only a conditional conclusion: either the volume bound above holds, or $M$ contains an embedded connected closed incompressible surface of genus 2 . The weakening of the lower bound on the dimension of homology in the hypothesis is made possible by the results of [6]. In the case where $M$ contains an embedded connected closed incompressible surface of genus 2 and $H_{1}\left(M ; \mathbb{Z}_{2}\right)$ has dimension at least 6 , a Dehn filling argument combined with Theorem 6.1 gives a better volume bound of 6.45 .

Theorem 6.3. Let $M$ be a closed, orientable hyperbolic 3-manifold with

$$
\operatorname{dim}_{\mathbb{Z}_{2}} H_{1}\left(M ; \mathbb{Z}_{2}\right) \geq 4
$$

and suppose that the image of the cup product map $H^{1}\left(M ; \mathbb{Z}_{2}\right) \otimes$ $H^{1}\left(M ; \mathbb{Z}_{2}\right) \rightarrow H^{2}\left(M ; \mathbb{Z}_{2}\right)$ has dimension at most 1 . Then $M$ has volume greater than 3.08 .

Theorem 6.3 should be compared with Theorem 3.1 of [6], which gives the same conclusion under the hypothesis that the $\mathbb{Z}_{2}$-homology of $M$ has dimension at least 6 , and with no restriction on cup product. As with that theorem, the proof of Theorem 6.3 uses the fact that if $\pi_{1}(M)$ is 3 -free, $M$ has volume greater than 3.08 (see Corollary 9.3 of [1]). If $\pi_{1}(M)$ has a 3-generator subgroup $G$ which is not free, the homological hypotheses of Theorem 6.3 ensure that $M$ has a two-sheeted cover $\widetilde{M}$ to which $G$ lifts, with $\operatorname{dim}_{\mathbb{Z}_{2}} H_{1}\left(\widetilde{M} ; \mathbb{Z}_{2}\right) \geq 6$. Then Theorem 5.1 of $[6]$ implies that $\widetilde{M}$ contains a connected closed incompressible surface of genus 2 , and Theorem 6.1 implies that $\widetilde{M}$ has volume greater than 6.45 , hence that $M$ has volume greater than 3.22 .

\section{Topological preliminaries}

In general we will follow [9] for standard terminology concerning 3-manifolds. (This includes, for example, the terms "irreducible" and "boundary irreducible" which were used in the Introduction.) Here we will explain a few special conventions and collect some preliminary results used throughout this paper.

We will work in the PL category in Sections 2-5, and in the smooth category in Section 6. The only result from the earlier sections quoted in Section 6 is Corollary 5.1, and the smooth version of this result follows 
from the PL version. We will also use, generally with explicit mention, the well-known fact that a closed, orientable hyperbolic 3-manifold is simple.

In Sections 2-5 we will use the following conventions concerning regular neighborhoods. Let $K$ be a compact polyhedron in a PL $n$-manifold $M$. We define a semi-regular neighborhood of $K$ in $M$ to be a neighborhood of $K$ which is a compact PL submanifold of $M$ and admits a polyhedral collapse to $K$. We define a regular neighborhood of $K$ in $M$ to be a semiregular neighborhood $N$ of $K$ in $M$ such that $N \cap \partial M$ is a semi-regular neighborhood of $K \cap \partial M$ in $\partial M$.

Let $Y$ be a subset of a topological space $X$, and suppose that $X$ and $Y$ are locally path connected. We will say that $Y$ is $\pi_{1}$-injective in $X$ if whenever $A$ and $B$ are components of $X$ and $Y$, respectively, such that $B \subset A$, the inclusion homomorphism $\pi_{1}(B) \rightarrow \pi_{1}(A)$ is injective. We will refer to $\bar{Y} \cap \overline{X-Y}$ as the frontier of $Y$ in $X$.

A closed orientable surface $S$ in the interior of an orientable 3-manifold $M$ will be termed incompressible if $S$ is $\pi_{1}$-injective in $M$ and no component of $S$ is a sphere. We shall not use the term "incompressible" for bounded surfaces.

We follow the conventions of [16] regarding Heegaard splittings and compression bodies. The following standard fact is a direct consequence of the definitions.

Lemma 2.1. Let $Q$ be an orientable 3-manifold with boundary, and suppose $\mathcal{S}$ is a Heegaard surface in $Q$.

(1) Let $Q^{\prime}$ be obtained from $Q$ by attaching a 2-handle to a component of $\partial Q$. Then $\mathcal{S}$ is a Heegaard surface in $Q^{\prime}$.

(2) Let $Q^{\prime}$ be obtained from $Q$ by attaching a handlebody to $Q$ along a component of $\partial Q$. Then $\mathcal{S}$ is a Heegaard surface in $Q^{\prime}$.

The lemma below is also standard, and will be used in Sections 3 and 5 .

Lemma 2.2. Let $Q$ be an orientable 3-manifold with boundary, and suppose $\mathcal{S}$ is a Heegaard surface in $Q$ of genus $g$. Let $Q^{\prime}$ be obtained from $Q$ by adding a 1-handle with both attaching disks in the same component of $Q-\mathcal{S}$. Then $\operatorname{Hg}\left(Q^{\prime}\right) \leq g+1$.

Proof. By definition we have $Q=C_{1} \cup C_{2}$ where $C_{1}$ and $C_{2}$ are compression bodies such that $\partial_{+} C_{1}=\mathcal{S}=\partial_{+} C_{2}$ and $C_{1} \cap C_{2}=\mathcal{S}$. After relabeling we may assume that $Q^{\prime}$ is obtained by attaching a 1 -handle $H$ to $\partial_{-} C_{1}$. We may write $C_{1}$ as $(\mathcal{S} \times I) \cup \mathcal{D} \cup \mathcal{T}$ where $\mathcal{S}$ is identified with $\mathcal{S} \times\{1\}, \mathcal{D}$ is 
a union of disjoint 2-handles attached along annuli in $\mathcal{S} \times\{0\}$, and $\mathcal{T}$ is a union of 3 -handles. Since $\mathcal{D} \cap \partial C_{1}$ is a union of disjoint disks, there is an ambient isotopy of $C_{1}$ which is constant on $\mathcal{S}$ and which moves the two attaching disks of $H$ so that they are disjoint from $\mathcal{D}$. We may thus assume that the attaching disks for $H$ are contained in $\mathcal{S} \times\{0\}$.

Let $N_{0}$ be a regular neighborhood in $H$ of its core. We have $N_{0} \cap \partial_{-} C_{1}=$ $(E \times\{0\}) \cup\left(E^{\prime} \times\{0\}\right)$, where $E$ and $E^{\prime}$ are disjoint disks in $\mathcal{S}=\partial_{+} C_{1}$. Let $N=N_{0} \cup(E \times I) \cup\left(E^{\prime} \times I\right)$, so that $E=E \times\{1\}$ and $E^{\prime}=E^{\prime} \times\{1\}$ are contained in $\partial N$. Set

$$
\mathcal{S}^{\prime}=\overline{\mathcal{S}-\left(E \cup E^{\prime}\right)} \cup \overline{\partial N-\left(E \cup E^{\prime}\right)} .
$$

The surface $\mathcal{S}^{\prime}$ has genus $g+1$ by construction. To complete the proof we will show that it is a Heegaard surface for $Q \cup H$.

Let $P=\left(\overline{\mathcal{S}-\left(E \cup E^{\prime}\right)} \times I\right) \cup \overline{H-N_{0}}$ and set $C_{1}^{\prime}=P \cup \mathcal{D}$. Note that $P$ is a semi-regular neighborhood of $\mathcal{S}^{\prime}$ in $C_{1}^{\prime}$, and hence homeomorphic to $\mathcal{S}^{\prime} \times I$. The attaching annuli of the 2-handles in $\mathcal{D}$ lie in the component of $\partial P$ which is disjoint from $\mathcal{S}^{\prime}$. It follows that $C_{1}^{\prime}$ is a compression body with $\partial_{+} C_{1}^{\prime}=\mathcal{S}^{\prime}$.

Next let $C_{2}^{\prime}=C_{2} \cup N$. From the dual description of $C_{2}$ as $\left(\partial_{-} C_{2}\right) \times I$ with a collection of 1-handles attached, it follows that $C_{2} \cup N$ is a compression body with $\partial_{+} C_{2}^{\prime}=\mathcal{S}^{\prime}$.

By construction we have $Q^{\prime}=C_{1}^{\prime} \cup C_{2}^{\prime}$ and $C_{1}^{\prime} \cap C_{2}^{\prime}=\partial_{+} C_{1}^{\prime}=\partial_{+} C_{2}^{\prime}=$ $\mathcal{S}^{\prime}$. Thus $\mathcal{S}^{\prime}$ is indeed a Heegaard surface for $Q^{\prime}$.

The following relatively straightforward result will be used in Sections 3 and 5 .

Proposition 2.1. Let $g \geq 2$ be an integer. Let $M$ be an irreducible, orientable 3-manifold which is $\left(g, \frac{g+1}{2}\right)$-small. Let $V$ be a compact, connected, irreducible 3-dimensional submanifold of $M$ which is $\pi_{1}$-injective. Suppose that either

(i) $\bar{\chi}(V)<g-1$, or

(ii) $\bar{\chi}(V) \leq g-1$ and $V$ is boundary-reducible.

Then $V$ is a handlebody.

Proof. Choose a properly embedded (possibly empty) submanifold $\mathcal{D}$ of $V$ such that 
(1) each component of $\mathcal{D}$ is an essential disk,

(2) no two components of $\mathcal{D}$ are parallel, and

(3) $\mathcal{D}$ is maximal among all properly embedded submanifolds of $V$ satisfying (1) and (2).

(Since $V$ is irreducible, (1) and (2) imply that the components of $\partial \mathcal{D}$ are non-trivial and pairwise non-parallel simple closed curves in $\partial V$; hence a submanifold $\mathcal{D}$ satisfying $(1)$ and $(2)$ has at most $3 \bar{\chi}(V)$ components, and hence a maximal submanifold with these properties exists.)

Let $N$ be a regular neighborhood of $\mathcal{D}$ in $V$, and set $Q=\overline{V-N}$. In order to complete the proof it suffices to show that every component of $Q$ is a ball.

Let us denote by $B_{1}, \ldots, B_{\nu}$ the components of $Q$ that are balls, and by $R_{1}, \ldots, R_{k}$ the remaining components of $Q$. A priori we have $k \geq 0$ and $\nu \geq 0$. We must show that $k=0$.

If $Q$ contains an essential disk $D$ we may assume after an isotopy that $D \cap N=\emptyset$; then $D \cup \mathcal{D}$ is a properly embedded submanifold of $V$ satisfying (1) and (2), a contradiction to the maximality of $\mathcal{D}$. This shows that $Q$ is boundary irreducible, so that $\partial Q$ is $\pi_{1}$-injective in $Q$. But $Q$ is $\pi_{1}$-injective in $V$ since every component of $\mathcal{D}$ is a disk, and $V$ is $\pi_{1}$-injective in $M$ by hypothesis. Hence:

2.1(a). $\partial Q$ is $\pi_{1}$-injective in $M$.

The manifold $Q$ is obtained from the irreducible $V$ by splitting along a collection of disjoint properly embedded disks. Hence:

2.1(b). Each component of $Q$ is irreducible.

It follows from 2.1(b) that each $R_{i}$ is irreducible. Since by definition no $R_{i}$ is a ball, we deduce:

2.1(c). No boundary component of any $R_{i}$ can be a sphere.

Let $n$ denote the number of components of $\mathcal{D}$, and observe that $\bar{\chi}(Q)=$ $\bar{\chi}(V)-n$. Next we note that by properties (1) and (2) of $\mathcal{D}$, each component of $Q$ which is a ball must contain at least three components of the frontier $F$ of $N$ in $V$. Since each component of $N$ contains exactly two components 
of $F$, the number $\nu$ of components of $Q$ that are balls is at most $2 n / 3$. Hence

$$
\begin{aligned}
\bar{\chi}(V)-n & =\bar{\chi}(Q) \\
& =-\nu+\sum_{i+1}^{k} \bar{\chi}\left(R_{i}\right) \\
& \geq-(2 n / 3)+\sum_{i+1}^{k} \bar{\chi}\left(R_{i}\right)
\end{aligned}
$$

so that

$$
\sum_{i+1}^{k} \bar{\chi}\left(R_{i}\right) \leq \bar{\chi}(V)-(n / 3) .
$$

If alternative (i) of the hypothesis holds we have $\bar{\chi}(V)<g-1$ and $n \geq 0$; if alternative (ii) holds, we have $\bar{\chi}(V) \leq g-1$ and $n>0$. Thus in either case, (2.1) implies that

$$
\sum_{i+1}^{k} \bar{\chi}\left(R_{i}\right)<g-1 .
$$

On the other hand, 2.1(c) implies that $\bar{\chi}\left(R_{i}\right) \geq 0$ for each $i$ with $1 \leq i \leq$ $k$. In view of $(2.2)$ it follows that

$$
\bar{\chi}\left(R_{i}\right)<g-1
$$

for each $i$ with $1 \leq i \leq k$.

We now proceed to the proof that $k=0$. Suppose that $k \geq 1$, and consider the manifold $R_{1}$. By 2.1 (a), the boundary of $R_{1}$ is $\pi_{1}$-injective in $M$. By 2.1(c), no component of $\partial R_{1}$ is a sphere. Hence every component of $\partial R_{1}$ is incompressible in $M$. Furthermore, we have

$$
\bar{\chi}\left(\partial R_{1}\right)=2 \bar{\chi}\left(R_{1}\right)<2 g-2
$$

by (2.3). If $\partial R_{1}$ is connected, it is a separating connected closed incompressible surface with $\bar{\chi}\left(\partial R_{1}\right)<2 g-2$, so that its genus is strictly less than $g$. This contradicts the hypothesis.

Now suppose that $\partial R_{1}$ is disconnected. Since $\bar{\chi}\left(\partial R_{1}\right)<2 g-2$ and no component of $\partial R_{1}$ is a sphere, we have $\bar{\chi}(S)<g-1$ for some component $S$ of $\partial R_{1}$. This means that $S$ is a connected closed incompressible surface of genus strictly less than $\frac{g+1}{2}$. Again we have a contradiction to the hypothesis. 


\section{Non-separating surfaces}

The purpose of this section is to prove Theorem 3.1, which was stated in the Introduction.

Definition 3.1. If a 3 -manifold $X$ has the structure of an $I$-bundle over a surface $T$ and $p: X \rightarrow T$ is the bundle projection, we will call $\partial_{v} X \doteq$ $p^{-1}(\partial T)$ the vertical boundary of $X$ and $\partial_{h} X \doteq \overline{\partial X-\partial_{v} X}$ the horizontal boundary of $X$.

Note that $\partial_{v} X$ inherits the structure of an $I$-bundle over $\partial T$, and $\partial_{h} X$ the structure of a $\partial I$-bundle over $T$, from the original $I$-bundle structure on $X$.

Theorem 3.1. Let $M$ be a closed, simple 3-manifold containing a nonseparating connected closed incompressible surface $S$ of genus $g$. Suppose that $\bar{\chi}(\operatorname{kish}(M \backslash \backslash S))<2 g-2$, and that $M$ is $(2 g-1, g)$-small. Then $\operatorname{Hg}(M) \leq$ $2 g+1$.

Proof. Let $M$ and $S$ be as in the statement of the theorem. Set $M^{\prime}=M \backslash \backslash S$, and note that $\bar{\chi}\left(M^{\prime}\right)=2 g-2$. Since by hypothesis we have $\bar{\chi}\left(\operatorname{kish}\left(M^{\prime}\right)\right)<$ $2 g-2$, the characteristic submanifold of $M^{\prime}$ has a component $X$ which is an $I$-bundle over a surface with negative Euler characteristic. We identify $M^{\prime}$ with $\overline{M-N}$, where $N$ is a regular neighborhood of $S$ in $M$; we then have $X \subset \overline{M-N} \subset M$. We set $\Sigma=N \cup X \subset M$. Since the horizontal boundary of $X$ has Euler characteristic $2 \chi(X)$, we have

$$
\bar{\chi}(\Sigma)=\bar{\chi}(N)+\bar{\chi}(X)-2 \bar{\chi}(X)=2 g-2-\bar{\chi}(X) .
$$

Since $\chi(X)<0$, it follows that $\bar{\chi}(\Sigma)<2 g-2$.

Set $K=\overline{M-\Sigma} \subset \overline{M-N}=M^{\prime}$. (It may happen that $K=\emptyset$.) Since $\partial K=\partial \Sigma$, we have $\bar{\chi}(K)=\bar{\chi}(\Sigma)$, and hence

$$
\bar{\chi}(K)<2 g-2 \text {. }
$$

Since the frontier components of $K$ in $M^{\prime}$ are essential annuli, $K$ is $\pi_{1}$-injective in $M^{\prime}$. The incompressibility of $S$ implies that $M^{\prime}=\overline{M-N}$ is $\pi_{1}$-injective in $M$. Hence: 
3.1(a). $K$ is $\pi_{1}$-injective in $M$.

Note also that $M^{\prime}$ is irreducible because the surface $S$ is incompressible in the irreducible 3 -manifold $M$. The manifold $K$ is a union of components of the manifold obtained by splitting $M^{\prime}$ along a collection of disjoint properly embedded annuli. Hence:

3.1(b). Every component of $K$ is irreducible.

Since each component of $K$ contains a component of the frontier of the characteristic submanifold of $M^{\prime}$, which is an essential annulus in $M^{\prime}$, no component of $K$ is a ball. In view of 3.1(b) it follows that no component of $\partial K$ is a sphere. Hence:

3.1(c). Every component of $K$ has non-positive Euler characteristic.

Now consider any component $V$ of $K$. Set $V^{\prime}=K-V$. It follows from $3.1(\mathrm{c})$ that $\bar{\chi}\left(V^{\prime}\right) \geq 0$. We have $\bar{\chi}(V)=\bar{\chi}(K)-\bar{\chi}\left(V^{\prime}\right)$, and hence by $(3.1)$

$$
\bar{\chi}(V)<2 g-2 .
$$

By hypothesis $M$ is $(2 g-1, g)$-small. Since $g=\frac{(2 g-1)+1}{2}$, this means that $M$ is $\left(2 g-1, \frac{(2 g-1)+1}{2}\right)$-small. In view of $3.1(\mathrm{a}), 3.1(\mathrm{~b})$ and $(3.2)$, case (i) of the hypothesis of Proposition 2.1 holds with $2 g-1$ playing the role of $g$. Proposition 2.1 therefore implies that $V$ is a handlebody.

Thus we have shown:

3.1(d). Every component of $K=\overline{M-\Sigma}$ is a handlebody.

We now turn to the estimation of $\operatorname{Hg}(M)$. First note that since $N$ is a trivial $I$-bundle over a surface of genus $g$, it can be obtained from a handlebody $J$ of genus $2 g$ by adding a 2-handle. The boundary $\mathcal{S}$ of a collar neighborhood of $\partial J$ in $J$ is a Heegaard surface of genus $2 g$ in $J$. Hence by assertion (1) of Lemma 2.1, $\mathcal{S}$ is a Heegaard surface in $N$. Note that $\partial N$ is contained in a single component of $N-\mathcal{S}$.

On the other hand, recall that $\Sigma=N \cup X$, where $X$ is an $I$-bundle over a connected surface $T$ and $N \cap X=\partial_{h} X$ is the horizontal boundary of $X$. Let $E \subset T$ be a disk such that for each boundary component $c$ of $T$, the set $E \cap c$ is a non-empty union of disjoint arcs in $c$. Let $p: X \rightarrow T$ denote the bundle projection, and set $Y=p^{-1}(E)$. Then $Y$ inherits the structure of a (necessarily trivial) $I$-bundle over $E$, and $Y \cap N=Y \cap \partial_{h} X$ is the horizontal 
boundary of $Y$, consisting of two disks. Thus the set $Y$ may be thought of as a 1-handle attached to the submanifold $N$. Since $\mathcal{S}$ is a genus- $2 g$ Heegaard surface in $N$, and $\partial N$ is contained in a single component of $N-\mathcal{S}$, it follows from Lemma 2.2 that $\operatorname{Hg}(N \cup Y) \leq 2 g+1$.

Next, note that each component of $\overline{(\partial T)-E}$ is an arc, and hence that each component of the set $\mathcal{D} \doteq p^{-1}(\overline{(\partial T)-E)})$ is a disk. Note also that $\mathcal{D} \cap$ $(N \cup Y)=\partial \mathcal{D}$. Hence if $R$ denotes a regular neighborhood of $\mathcal{D}$ relative to $\overline{X-Y}$, saturated in the fibration of $X$, the manifold $N \cup Y \cup R$ is obtained from $N \cup Y$ by adding finitely many 2-handles. By assertion (1) of Lemma 2.1 it follows that

$$
\operatorname{Hg}(N \cup Y \cup R) \leq \operatorname{Hg}(N \cup Y) \leq 2 g+1
$$

Finally, note that each component of $\overline{M-(N \cup Y \cup R)}$ is either

(a) a component of $\overline{X-(Y \cup R)}$ or

(b) a component of $\overline{M-\Sigma}$.

Each component of type (a) is a sub-bundle of $X$ over a bounded subsurface, and is therefore a handlebody. Each component of type (b) is a handlebody by virtue of 3.1(d). Since each component of $\overline{M-(N \cup Y \cup R)}$ is a handlebody, it now follows from assertion (2) of Lemma 2.1 that

$$
\operatorname{Hg}(M) \leq \operatorname{Hg}(N \cup Y \cup R) \leq 2 g+1
$$

\section{Annulus bodies and shallow manifolds}

In the next two sections, we develop an organizing principle for cylinders properly embedded in the complement of a separating connected closed incompressible surface.

Definition 4.1. Let $Y$ be a compact, connected 3-manifold, and let $S$ be a (possibly disconnected) closed, 2-dimensional submanifold of $\partial Y$. We shall say that $Y$ is an annulus body relative to $S$ if there is a properly embedded annulus $A \subset Y$ with $\partial A \subset S$, such that $Y$ is a semi-regular neighborhood of $S \cup A$.

Lemma 4.1. Let $Y$ be a compact, connected 3-manifold, and let $S \subset \partial Y$ be a closed 2-manifold. If $Y$ is an annulus body relative to $S$, then $Y$ is also an annulus body relative to $(\partial Y)-S$. Furthermore, we have $\bar{\chi}(Y)=\bar{\chi}(S)=$ $\bar{\chi}((\partial Y)-S)$. 
Proof. We set $T=(\partial Y)-S$.

By the definition of an annulus body, $Y$ is a semi-regular neighborhood of $S \cup A$ for some properly embedded annulus $A \subset Y$ with $\partial A \subset S$. Let $R$ be a regular neighborhood of $A$ in $Y$. Then there is a PL homeomorphism $j$ : $S^{1} \times[-1,1] \times[-1,1] \rightarrow R$ such that $j\left(S^{1} \times\{0\} \times[-1,1]\right)=A$ and $j\left(S^{1} \times\right.$ $[-1,1] \times\{-1,1\})=R \cap S$. Let $B$ denote the annulus $j\left(S^{1} \times[-1,1] \times\{0\}\right)$. Set $Q=\overline{Y-R}$, and let $N$ be a regular neighborhood of $Q \cap S$ in $Q$, chosen small enough so that $N \cap B=\emptyset$. Set $Y^{\prime}=N \cup R$. Then $Y^{\prime}$ is a compact 3-manifold and $S \subset \partial Y^{\prime}$. If we set $T^{\prime}=\left(\partial Y^{\prime}\right)-S$, then the annulus $B$ is properly embedded in $Y^{\prime}$ and $\partial B \subset T^{\prime}$. Furthermore, $Y^{\prime}$ is a semi-regular neighborhood of $T^{\prime} \cup B$, and hence $Y^{\prime}$ is an annulus body relative to $T^{\prime}$.

On the other hand, $Y$ and $Y^{\prime} \subset Y$ are both semi-regular neighborhoods of $S \cup A$, and $Y^{\prime} \cap \partial Y=S$. Hence $\overline{Y-Y^{\prime}}$ is a collar neighborhood of $T \subset$ $\partial Y$ in $Y$. In particular the pairs $(Y, T)$ and $\left(Y^{\prime}, T^{\prime}\right)$ are PL homeomorphic, and so $Y$ is an annulus body relative to $T$.

To prove the second assertion, we note that since $Y$ and $S \cup A$ are homotopy equivalent, we have $\chi(Y)=\chi(S \cup A)$; and that since $A$ and $A \cap S=\partial A$ have Euler characteristic 0, we have $\chi(S \cup A)=\chi(S)$. This proves that $\bar{\chi}(S)=\bar{\chi}(Y)$. Since we have shown that $Y$ is also an annulus body relative to $T$, we may substitute $T$ for $S$ in the last equality and conclude that $\bar{\chi}(T)=\bar{\chi}(Y)$.

Definition 4.2. Let $Z$ be a compact, connected, orientable 3-manifold, and let $S \subset \partial Z$ be a closed surface. We will say that $Z$ is shallow relative to $S$ if $Z$ may be written in the form $Z=Y \cup J$, where $Y \supset S$ and $J$ are compact 3-dimensional submanifolds of $Z$ such that

(1) each component of $J$ is a handlebody,

(2) $Y$ is an annulus body relative to $S$,

(3) $Y \cap J=\partial J$, and

(4) $\partial J$ is a union of components of $(\partial Y)-S$.

(The submanifold $J$ may be empty.)

Lemma 4.2. Let $Q$ be a compact orientable 3-manifold, let $Z \subset Q$ be a compact submanifold whose frontier $S$ is a connected closed surface in int $Q$, and suppose that $Z$ is shallow relative to $S$. Then $\operatorname{Hg}(Q) \leq 1+\operatorname{Hg}(\overline{Q-Z})$.

Proof. We set $Q_{0}=\overline{Q-Z}$ and $g=\operatorname{Hg}\left(Q_{0}\right)$. We write $Z=Y \cup J$, where $Y$ and $J$ satisfy conditions (1)-(4) of Definition 4.2. Since $Y$ is an annulus body 
relative to $S$, it follows from Definition 4.1 that there is a properly embedded annulus $A \subset Y$ with $\partial A \subset S$, such that $Y$ is a semi-regular neighborhood, relative to $Y$ itself, of $S \cup A$.

Let $\alpha$ denote a co-core of the annulus $A$, and fix a regular neighborhood $h$ of $\alpha$ in $Y$ such that $h \cap A$ is a regular neighborhood of $\alpha$ in $A$. The manifold $Q_{0} \cup h$ is obtained from $Q_{0}$ by attaching a 1-handle that has both its attaching disks in the component $S$ of $\partial Q_{0}$. Hence it follows from Lemma 2.2 that $\operatorname{Hg}\left(Q_{0} \cup h\right) \leq 1+\operatorname{Hg}\left(Q_{0}\right)=1+g$.

The disk $D=\overline{A-(h \cap A)}$ is properly embedded in the manifold $\overline{Y-h}$. Hence if $R$ denotes a regular neighborhood of $D$ relative to $\overline{Y-h}$, the manifold $X_{0} \doteq Q_{0} \cup h \cup R$ is obtained from $Q_{0} \cup h$ by attaching a 2-handle. It therefore follows from assertion (1) of Lemma 2.1 that $\operatorname{Hg}\left(X_{0}\right) \leq$ $\operatorname{Hg}\left(Q_{0} \cup h\right) \leq 1+g$. But $X \doteq Q_{0} \cup Y$ is a semi-regular neighborhood of $X_{0}$ relative to $X$ itself, and is therefore homeomorphic to $X_{0}$. Hence $\operatorname{Hg}(X) \leq$ $1+g$.

We have $Q=X \cup J$. In view of conditions (1), (3) and (4) of Definition 4.2, it follows that each component of $\overline{Q-X}$ is a handlebody whose boundary is contained in $\partial X$. From assertion (2) of Lemma 2.1 we deduce that $\operatorname{Hg}(Q) \leq \operatorname{Hg}(X) \leq 1+g$.

Lemma 4.3. Suppose that $Z$ is a compact, connected, orientable 3-manifold, that $\partial Z$ is connected, and that $Z$ is shallow relative to $\partial Z$. Let $g$ denote the genus of $\partial Z$. Then $\operatorname{Hg}(Z) \leq g+1$.

Proof. Let $N$ be a boundary collar for $Z$. Then $N$ has a Heegaard splitting of genus $g$, the frontier $S$ of $N$ in $Z$ is connected, and $\overline{Z-N}$ is shallow relative to $S$. The result therefore follows upon applying Lemma 4.2, with $Z$ and $\overline{Z-N}$ playing the respective roles of $Q$ and $Z$ in that lemma.

Lemma 4.4. Let $g \geq 2$ be an integer. Let $Z$ be a compact, orientable 3manifold having exactly two boundary components $S_{0}$ and $S_{1}$, both of genus $g$. Then $Z$ is shallow relative to $S_{0}$ if and only if either

(i) $Z$ is an annulus body relative to $S_{0}$, or

(ii) there is a solid torus $K \subset Z$ such that $K \cap \partial Z$ is an annulus contained in $S_{1}$, and the pair $\left(\overline{Z-K}, S_{0}\right)$ is homeomorphic to $\left(S_{0} \times I, S_{0} \times\{0\}\right)$.

Proof. If alternative (i) holds then $Z$ is shallow relative to $S_{0}$ : it suffices to take $Y=Z$ and $J=\emptyset$ in Definition 4.2. If alternative (ii) holds, let $J$ be a regular neighborhood in int $K$ of a core curve of $K$ and set $Y=\overline{Z-J}$. Then $Y$ is an annulus body relative to $S_{1}$. (The annulus $A$ appearing in 
Definition 4.1 is bounded by two parallel simple closed curves.) It now follows from Definition 4.2 that $Z$ is shallow relative to $S_{0}$.

Conversely, suppose that $Z$ is shallow relative to $S_{0}$. Let us write $Z=$ $Y \cup J$, where $Y$ and $J$ satisfy conditions (1)-(4) of Definition 4.2, with $S_{0}$ playing the role of $S$. Set $T=(\partial Y)-S_{0}$. Since $Y$ is an annulus body relative to $S_{0}$, it follows from Lemma 4.1 that $Y$ is an annulus body relative to $T$. This means that $Y$ is a semi-regular neighborhood of $T \cup A$, where $A$ is an annulus with $A \cap T=\partial A$. Since $Y$ is connected it follows that $T$ has at most two components.

The conditions of Definition 4.2 imply that $T$ is the disjoint union of $\partial J$ with $S_{1}$. Since $T$ has at most two components and $S_{1}$ has exactly one component, $\partial J$ has at most one component. If $\partial J=\emptyset$ then $J=\emptyset$, i.e. $Z=Y$. This implies alternative (i) of the present lemma.

Now consider the case in which $\partial J$ has exactly one component. In this case $J$ is a single handlebody, and $\partial J$ and $S_{1}$ are the components of $T$. According to Lemma 4.1 we have

$$
2 g-2=\bar{\chi}\left(S_{0}\right)=\bar{\chi}(T)=\bar{\chi}\left(S_{1}\right)+\bar{\chi}(\partial J) .
$$

But since $S_{1}$ is a surface of genus $g$ we have $\bar{\chi}\left(S_{1}\right)=2 g-2$, and hence $\bar{\chi}(J)=0$. Thus $J$ is a solid torus.

Now $Y$ is a semi-regular neighborhood of $T \cup A=S_{1} \cup A \cup \partial J$. Since $Y$ is connected, $A$ must have one boundary component in $S_{1}$ and one in $\partial J$. Let $R$ be a regular neighborhood of $A$ in $Y$. Then $R$ is a solid torus meeting $S_{1}$ and $\partial J$, respectively, in regular neighborhoods of the simple closed curves $A \cap S_{1}$ and $A \cap \partial J$. Let $K=J \cup R$. Since $J$ is a solid torus and $A \cap J$ is parallel in $R$ to its core, $K$ is a solid torus. Furthermore, $K \cap S_{1}=R \cap S_{1}$ is an annulus.

Now set $Q=\overline{Y-R}=\overline{Z-K}$. If $N$ is a regular neighborhood in $Q$ of $Q \cap T$, then $Y^{\prime}=N \cup R$ is a semi-regular neighborhood of $T \cup A$ contained in $Y$. Therefore $\overline{Y-Y^{\prime}}$ is a collar neighborhood of $S_{0}$ in $Y$; that is, the pairs $\left(\overline{Y-Y^{\prime}}, S_{0}\right)$ and $\left(S_{0} \times I, S_{0} \times\{0\}\right)$ are homeomorphic. But by the definition of $N$, the pair $\left(\overline{Y-Y^{\prime}}, S_{0}\right)=\left(\overline{Q-N}, S_{0}\right)$ is homeomorphic to $\left(Q, S_{0}\right)=\left(\overline{Z-K}, S_{0}\right)$, and alternative (ii) of the present lemma holds in this case.

Lemma 4.5. Let $g \geq 2$ be an integer. Let $Z$ be a compact, orientable 3manifold having exactly two boundary components $S_{0}$ and $S_{1}$, both of genus $g$. Then $Z$ is shallow relative to $S_{0}$ if and only if it is shallow relative to $S_{1}$. 
Proof. By symmetry it suffices to show that if $Z$ is shallow relative to $S_{0}$ then it is shallow relative to $S_{1}$. In view of Lemma 4.4, it suffices to show that if one of the alternatives (i) and (ii) of that lemma holds, then it still holds when $S_{0}$ is replaced by $S_{1}$. For alternative (i) this follows from Lemma 4.1. Now suppose that alternative (ii) of Lemma 4.4 holds. Let $c$ be a core curve of the annulus $\overline{\partial K-(K \cap \partial Z)}$. Since $\left(\overline{Z-K}, S_{0}\right)$ is homeomorphic to $\left(S_{0} \times I, S_{0} \times\{0\}\right)$, there is a properly embedded annulus $\alpha \subset \overline{Z-K}$ joining $c$ to a simple closed curve in $S_{0}$. Let $B$ be a regular neighborhood of $K \cup$ $\alpha$ in $Z$. Set $P=\overline{Z-B}$, choose a regular neighborhood $N$ of $P \cup S_{1}$ in $Z$, and set $K^{\prime}=\overline{Z-N}$. Then $K^{\prime}$ is a solid torus, $K^{\prime} \cap \partial Z$ is an annulus contained in $S_{0}$, and the pair $\left(\overline{Z-K^{\prime}}, S_{1}\right)=\left(N, S_{1}\right)$ is homeomorphic to $\left(S_{1} \times I, S_{1} \times\{0\}\right)$.

\section{Separating surfaces}

5.1. In this section we will use the theory of books of $I$-bundles as developed in [1]. We recall the definition here, in a slight paraphrase of the form given in $[1]$.

A book of $I$-bundles is a triple $\mathcal{W}=(W, \mathcal{B}, \mathcal{P})$, where $W$ is a (possibly disconnected) compact, orientable 3 -manifold, and $\mathcal{B}, \mathcal{P} \subset W$ are submanifolds such that

- each component of $\mathcal{B}$ is a solid torus;

- $\mathcal{P}$ is an $I$-bundle over a (possibly disconnected) 2-manifold, and every component of $\mathcal{P}$ has Euler characteristic $\leq 0$;

- $W=\mathcal{B} \cup \mathcal{P}$;

- $\mathcal{B} \cap \mathcal{P}$ is the vertical boundary of $\mathcal{P}$;

- $\mathcal{B} \cap \mathcal{P}$ is $\pi_{1}$-injective in $\mathcal{B}$; and

- each component of $\mathcal{B}$ meets at least one component of $\mathcal{P}$.

As in [1], we shall denote $W, \mathcal{B}$ and $\mathcal{P}$ by $|\mathcal{W}|, \mathcal{B}_{\mathcal{W}}$ and $\mathcal{P}_{\mathcal{W}}$, respectively. The components of $\mathcal{B}_{\mathcal{W}}$ will be called bindings of $\mathcal{W}$, and the components of $\mathcal{P}_{\mathcal{W}}$ will be called its pages. The submanifold $\mathcal{B} \cap \mathcal{P}$, whose components are properly embedded annuli in $W$, will be denoted $\mathcal{A}_{\mathcal{W}}$.

An important observation, which follows from the definitions, is that if $W$ is a simple 3-manifold with kish $W=\emptyset$, then $W=|\mathcal{W}|$ for some book of $I$-bundles $\mathcal{W}$. 
Lemma 5.1. If $\mathcal{W}$ is any connected book of I-bundles then $\mathcal{A}_{\mathcal{W}}$ is $\pi_{1}$ injective in $|\mathcal{W}|$. Furthermore, $|\mathcal{W}|$ is an irreducible 3-manifold.

Proof. If $A$ is any component of $\mathcal{A}=\mathcal{A}_{\mathcal{W}}$, then $A$ lies in the frontier of a unique component $P$ of $\mathcal{P}=\mathcal{P}_{\mathcal{W}}$ and in a unique component $B$ of $\mathcal{B}=\mathcal{B}_{\mathcal{W}}$. Since $A$ is an annulus of non-zero degree in the solid torus $B$, it is $\pi_{1}$-injective in $B$. It is also $\pi_{1}$-injective in $P$, since $A$ is a vertical boundary annulus of the $I$-bundle $P$ and $\chi(P) \leq 0$. It follows that $\mathcal{A}$ is $\pi_{1}$-injective in $\mathcal{B}$ and in $\mathcal{P}$ and hence in $W=|\mathcal{W}|$, which is the first assertion.

To prove the second assertion, we note that $\mathcal{B}$ is irreducible because its components are solid tori, and that $\mathcal{P}$ is irreducible because its components are $I$-bundles over surfaces of Euler characteristic $\leq 0$. Thus $W$ contains the $\pi_{1}$-injective, two-sided, properly embedded 2-manifold $\mathcal{A}$, and the manifold obtained by splitting $W$ along $\mathcal{A}$ is irreducible. It follows that $W$ is itself irreducible.

Lemma 5.2. Let $M$ be a closed simple 3-manifold. Suppose that $\mathcal{W}$ is a connected book of I-bundles with $W=|\mathcal{W}| \subset M$, and that $\partial W$ is a connected incompressible surface in $M$. Let $g$ denote the genus of $\partial W$. Suppose that $M$ is $\left(g, \frac{g+1}{2}\right)$-small. Then $W$ is shallow relative to $\partial W$.

Proof. We first consider the degenerate case in which $\mathcal{W}$ has no bindings, so that $W$ is an $I$-bundle over a closed surface $T$ with $\chi(T)=2-2 g<0$. We choose an orientation-preserving simple closed curve $C \subset T$ and let $A$ denote the annulus $p^{-1}(C)$, where $p$ is the bundle projection. If $Y$ denotes a regular neighborhood of $(\partial W) \cup A$, then $\overline{W-Y}$ is homeomorphic to an $I$-bundle over a bounded surface, and hence to a handlebody; hence $W$ is shallow in this case.

Now assume that $\mathcal{W}$ has at least one binding, so that every page is an $I$-bundle over a bounded surface. The sum of the Euler characteristics of the pages of $\mathcal{W}$ is equal to $\chi(W)=1-g<0$. In particular, $\mathcal{W}$ has a page $P_{0}$ with $\chi\left(P_{0}\right)<0$. Then $P_{0}$ is an $I$-bundle over some base surface $T$; we let $p: P_{0} \rightarrow T$ denote the bundle projection. We choose a component $C$ of $\partial T$ and set $A=p^{-1}(C) \subset \partial_{v} P_{0}$. (See Definition 3.1.) Since $\chi(T)=\chi\left(P_{0}\right)<0$, there is an arc $\alpha \subset T$ such that $\partial \alpha=\alpha \cap \partial T \subset C$, and $\alpha$ is not parallel in $T$ to an arc in $C$. Now $A$ is a properly embedded annulus in $W$, and $D=p^{-1}(\alpha)$ is a properly embedded disk in $P_{0}$. The boundary of $D$ consists of two vertical arcs in $A \subset \partial_{v} P_{0}$, and of two properly embedded arcs in $\partial_{h} A_{0}$, each of which projects to $\alpha$ under the bundle projection. Since $\alpha$ is not parallel in $T$ to an arc in $C$, each of the four arcs comprising $\partial D$ 
is essential in either $\partial_{h} P_{0}$ or $A$. Hence $\partial D$ is homotopically non-trivial in $A \cup \partial_{h} P_{0}$.

We set $X=W \backslash \backslash A$. We may identify $P_{0}$ with a submanifold of $X$. Since $\partial D \subset A \cup \partial_{h} P_{0}$, the disk $D$ is properly embedded in $X$. Each component of $\partial_{v} P_{0}$ is an annulus in $\mathcal{A}_{\mathcal{W}}$ by definition. Since the frontier curves of $A \cup \partial_{h} P_{0}$ in $\partial X$ are boundary components of such annuli, it follows from Lemma 5.1 that they are homotopically non-trivial in $X$. Therefore $A \cup \partial_{h} P_{0}$ is $\pi_{1^{-}}$ injective in $\partial X$. Since $\partial D$ is homotopically non-trivial in $A \cup \partial_{h} P_{0}$, it is homotopically non-trivial in $\partial X$. Hence the disk $D$ is essential in $X$.

Note that $\partial X$ may have either one or two components. In either case we have $\bar{\chi}(X)=\bar{\chi}(W)=g-1$.

The manifold $W$ is irreducible by Lemma 5.1, and $X$ is obtained by splitting $W$ along a properly embedded surface. Hence:

5.2(a). Each component of $X$ is irreducible.

We shall identify $X$ homeomorphically with $\overline{W-N}$, where $N$ is a regular neighborhood of $A$ in $W$. With this identification, it follows from Lemma 5.1 that $X$ is $\pi_{1}$-injective in $W$. On the other hand, the incompressibility of $\partial W$ implies that $W$ is $\pi_{1}$-injective in $M$. Hence:

5.2(b). $X$ is $\pi_{1}$-injective in $M$.

We now claim:

5.2(c). Each component of $X$ is a handlebody.

To prove $5.2(\mathrm{c})$, we recall that by hypothesis $M$ is $\left(g, \frac{g+1}{2}\right)$-small. Furthermore each component of $X$ is $\pi_{1}$-injective in $M$ by $5.2(\mathrm{~b})$, and is irreducible by $5.2(\mathrm{a})$. Hence it suffices to show that one of the conditions (i) or (ii) of Proposition 2.1 holds for each component $V$ of $X$.

If $X$ is connected, then it is boundary reducible since it contains the essential disk $D$. Since $\bar{\chi}(X)=g-1$, condition (i) of Proposition 2.1 holds with $V=X$.

Now suppose that $X$ has two components $X_{0}$ and $X_{1}$. Each of these is a union of pages and bindings of $\mathcal{W}$, and we may suppose them to be indexed so that $P_{0} \subset X_{0}$. For $i=0,1$ we have $\bar{\chi}\left(X_{i}\right)=\sum \bar{\chi}(P)$, where $P$ ranges over the pages contained in $X_{i}$. By the definition of a book of $I$-bundles, each term $\bar{\chi}(P)$ is non-negative. In particular we have $\bar{\chi}\left(X_{1}\right) \geq 0$, and since 
$\bar{\chi}\left(P_{0}\right)>0$ we have $\bar{\chi}\left(X_{0}\right)>0$. On the other hand, we have

$$
\bar{\chi}\left(X_{0}\right)+\bar{\chi}\left(X_{1}\right)=\bar{\chi}(X)=g-1
$$

It follows that $\bar{\chi}\left(X_{1}\right)<g-1$, so that condition (i) of Proposition 2.1 holds with $V=X_{1}$. On the other hand, we have $\bar{\chi}\left(X_{0}\right) \leq g-1$, and $X_{0}$ is boundary reducible since it contains the essential disk $D$. Thus condition (i) of Proposition 2.1 holds with $V=X_{0}$. This proves $5.2(\mathrm{c})$.

If $Y$ denotes a regular neighborhood of $(\partial W) \cup A$, then $Y$ is an annulus body relative to $\partial W$, and $\overline{W-Y}$ is homeomorphic to $X$ and is therefore a handlebody. By Definition 4.2 it follows that $W$ is shallow.

Definition 5.1. Let $M$ be a closed orientable 3-manifold, and let $g$ be an integer $\geq 2$. We define a $g$-layering to be a finite sequence $\left(Z_{0}, S_{1}, Z_{1}, \ldots, S_{n}\right.$, $\left.Z_{n}\right)$, where

- $n$ is a strictly positive integer,

- $S_{1}, \ldots, S_{n}$ are disjoint separating incompressible surfaces in $M$ with genus $g$,

- $Z_{0}, Z_{1}, \ldots, Z_{n}$ are the closures of the components of $M-\left(S_{1} \cup \cdots \cup\right.$ $\left.S_{n}\right)$

- $\partial Z_{0}=S_{1}, \partial Z_{n}=S_{n}$, and $\partial Z_{i}=S_{i} \sqcup S_{i+1}$ for $0<i<n$, and

- for $0<i<n, Z_{i}$ is shallow relative to $S_{i}$ and is not homeomorphic to $S_{i} \times I$.

We shall call the integer $n$ the depth of the $g$-layering $\left(Z_{0}, S_{1}, Z_{1}, \ldots, S_{n}, Z_{n}\right)$. We will say that a $g$-layering $\left(Z_{0}^{\prime}, S_{1}^{\prime}, Z_{1}^{\prime}, \ldots, S_{n^{\prime}}^{\prime}, Z_{n^{\prime}}^{\prime}\right)$ is a (strict) refinement of $\left(Z_{0}, S_{1}, Z_{1}, \ldots, S_{n}, Z_{n}\right)$ if $\left(S_{1}, \ldots, S_{n}\right)$ is a (proper) subsequence of the finite sequence $\left(S_{1}^{\prime}, \ldots, S_{n^{\prime}}^{\prime}\right)$. A $g$-layering will be called maximal if it has no strict refinement.

Lemma 5.3. Let $M$ be a closed orientable 3-manifold, and let $g$ be an integer $\geq 2$. If $\left(Z_{0}, S_{1}, Z_{1}, \ldots, S_{n}, Z_{n}\right)$ is a g-layering, then $\left(Z_{n}, S_{n}, Z_{n-1}, \ldots, S_{1}\right.$, $\left.Z_{0}\right)$ is also a g-layering. Furthermore, if $\left(Z_{0}, S_{1}, Z_{1}, \ldots, S_{n}, Z_{n}\right)$ is maximal, then $\left(Z_{n}, S_{n}, Z_{n-1}, \ldots, S_{1}, Z_{0}\right)$ is also maximal.

Proof. If $\left(Z_{0}, S_{1}, Z_{1}, \ldots, S_{n}, Z_{n}\right)$ is a $g$-layering, then for $0<i<n$, since $Z_{i}$ is shallow relative to $S_{i}$, it follows from Lemma 4.5 that $Z_{i}$ is shallow relative to $S_{i+1}$. This implies the first assertion. 
To prove the second assertion, suppose that $\left(Z_{n}, S_{n}, Z_{n-1}, \ldots, S_{1}, Z_{0}\right)$ is not maximal, so that it has a strict refinement $\left(Z_{n^{\prime}}^{\prime}, S_{n^{\prime}}^{\prime}, Z_{n^{\prime}-1}^{\prime}, \ldots, S_{1}^{\prime}, Z_{0}^{\prime}\right)$. Then $\left(Z_{0}^{\prime}, S_{1}^{\prime}, \ldots, Z_{n-1}^{\prime}, S_{n}^{\prime}, Z_{n}^{\prime}\right)$ is a $g$-layering according to the first assertion, and is a strict refinement of $\left(Z_{0}, S_{1}, \ldots, Z_{n-1}, S_{n}, Z_{n}\right)$. Hence $\left(Z_{0}, S_{1}, \ldots, Z_{n-1}, S_{n}, Z_{n}\right)$ is not maximal.

Lemma 5.4. Let $M$ be a closed orientable 3-manifold, and let $g$ be an integer $\geq 2$. Suppose that $M$ is $\left(g, \frac{g}{2}+1\right)$-small. If $\left(Z_{0}, S_{1}, Z_{1}, \ldots, S_{n}, Z_{n}\right)$ is a maximal g-layering, then $Z_{n}$ is either shallow relative to $S_{n}$ or acylindrical.

Proof. Let us suppose that $Z_{n}$ is not acylindrical. Let $A$ be an essential annulus in $Z_{n}$. We set $X=Z_{n} \backslash \backslash A$. We shall identify $X$ homeomorphically with $\overline{Z_{n}-N}$, where $N$ is a regular neighborhood of $A$ in $Z_{n}$.

If $Y$ denotes a regular neighborhood of $\left(S_{n}\right) \cup A$, then $Y$ is an annulus body relative to $S_{n}$, and $\bar{Z}_{n}-Y$ is ambiently isotopic to $X$. Hence in order to show that $Z_{n}$ is shallow, it suffices to show that each component of $\overline{Z_{n}-Y}$ is a handlebody.

Note that $\partial X$ has at most two components, and hence that $X$ has at most two components. We have

$$
g-1=\bar{\chi}\left(Z_{n}\right)=\bar{\chi}(X)=\sum_{V} \bar{\chi}(V)
$$

where $V$ ranges over the components of $X$. The essentiality of $A$ implies that $\bar{\chi}(F) \geq 0$ for each component $F$ of $\partial X$, and hence that $\bar{\chi}(V) \geq 0$ for each component $V$ of $\partial X$. It therefore follows from (5.1) that $\bar{\chi}(V) \leq g-1$ for each component $V$ of $X$.

Since $A$ is an essential annulus, $X$ is $\pi_{1}$-injective in $Z_{n}$. On the other hand, the incompressibility of $S_{n}$ implies that $Z_{n}$ is $\pi_{1}$-injective in $M$. Hence:

5.4(a). $X$ is $\pi_{1}$-injective in $M$.

Since $M$ is irreducible, the incompressibility of $S_{n}$ implies that $Z_{n}$ is irreducible. Since $A$ is a properly embedded annulus in $Z_{n}$, we deduce:

5.4(b). Each component of $X$ is irreducible.

We now claim:

5.4(c). For each component $V$ of $X$, some component of $\partial V$ is compressible in $M$. 
To prove 5.4(c), we first consider the case in which $\partial V$ is disconnected. In this case, since $\bar{\chi}(\partial V)=2 \bar{\chi}(V) \leq 2 g-2$, there is a component $F$ of $\partial V$ with $\bar{\chi}(F)<g$; hence the genus of $F$ is strictly less than $\frac{g}{2}+1$. Since $M$ is $\left(g, \frac{g}{2}+1\right)$-small, the surface $F$ must be compressible.

We next consider the case in which $\partial V$ is connected and $\bar{\chi}(V)<g-1$. In this case we have $\bar{\chi}(\partial V)<2 g-2$, so that $\partial V$ has genus strictly less than $g$. Furthermore, $\partial V$ separates $M$. Since $M$ is $\left(g, \frac{g}{2}+1\right)$-small, the surface $\partial V$ must be compressible.

There remains the case in which $\partial V$ is connected and $\bar{\chi}(V)=g-1$. In this case we set $S=\partial V$ and observe that $S$ is a separating surface of genus $g$. We shall assume that $S$ is incompressible and derive a contradiction. Since $X^{\prime} \doteq \overline{Z_{n}-Y}$ is ambiently isotopic to $X$, some component $V^{\prime}$ of $X^{\prime}$ is ambiently isotopic to $V$, and so $S^{\prime} \doteq \partial V^{\prime}$ is a separating connected closed incompressible surface of genus $g$.

We distinguish two subcases, depending on whether (a) $V$ is the only component of $X$, or (b) $X$ has a second component $U$. In subcase (a), the boundary components of $Y$ are $S_{n}$ and $S^{\prime}$. Since $Y$ is an annulus body relative to $S_{n}$, it is in particular shallow relative to $S_{n}$. Furthermore, $Y$ cannot be homeomorphic to $S_{n} \times I$, because it contains the annulus $A$, which is essential in $Z_{n}$ - and hence in $Y$ - and has its boundary contained in $S_{n}$. It now follows from Definition 5.1 that $\left(Z_{0}, S_{1}, Z_{1}, \ldots, S_{n}, Y, S^{\prime}, X^{\prime}\right)$ is a $g$-layering. This contradicts the maximality of $\left(Z_{0}, S_{1}, Z_{1}, \ldots, S_{n}, Z_{n}\right)$.

In subcase (b), it follows from (5.1) that $\bar{\chi}(U)=0$. Since $\partial X$ has at most two components, $\partial U$ is a single torus. The simplicity of $M$ implies that $\partial U$ is compressible in $M$, and since $U$ is $\pi_{1}$-injective in $M$ by $5.4(\mathrm{a}), \partial U$ cannot be $\pi_{1}$-injective in $U$. As $U$ is irreducible by $5.4(\mathrm{~b})$, it now follows that $U$ is a solid torus. Hence the component $U^{\prime}$ of $X^{\prime}$ which is ambiently isotopic to $U$ is a solid torus. According to Definition 4.2, this implies that $Z \doteq Y \cup U^{\prime}$ is shallow. The boundary components of $Z$ are $S_{n}$ and $S^{\prime}$. The shallow manifold $Z$ cannot be homeomorphic to $S_{n} \times I$, because it contains the annulus $A$, which is essential in $Z_{n}$ - and hence in $Z$ - and has its boundary contained in $S_{n}$. It now follows from Definition 5.1 that $\left(Z_{0}, S_{1}, Z_{1}, \ldots, S_{n}, Z, S^{\prime}, V^{\prime}\right)$ is a $g$-layering. This contradicts the maximality of $\left(Z_{0}, S_{1}, Z_{1}, \ldots, S_{n}, Z_{n}\right)$.

This completes the proof of $5.4(\mathrm{c})$.

Next we claim:

5.4(d). Each component of $X$ is boundary-reducible.

In fact, if a component $V$ of $X$ were boundary irreducible, then $\partial V$ would be $\pi_{1}$-injective in $V$. In view of 5.4 (a) it would follow that $\partial V$ is $\pi_{1}$-injective in $M$. But this contradicts 5.4(c). Thus 5.4(d) is established. 
We now turn to the proof that each component of $X$ is a handlebody, which will complete the proof of the lemma.

Let $V$ be any component of $X$. We have observed that $\bar{\chi}(V) \leq g-1$. By 5.4(a), $V$ is $\pi_{1}$-injective in $M$, by $5.4(\mathrm{~b})$ it is irreducible, and by $5.4(\mathrm{~d})$ it is boundary-reducible. Since the hypothesis implies in particular that $M$ is $\left(g, \frac{g+1}{2}\right)$-small, case (ii) of the hypothesis of Proposition 2.1 holds. It therefore follows from Proposition 2.1 that $V$ is a handlebody.

Lemma 5.5. Let $M$ be a closed orientable 3-manifold, and let $g$ be an integer $\geq 2$. Suppose that $M$ is $\left(g, \frac{g}{2}+1\right)$-small. If $\left(Z_{0}, S_{1}, Z_{1}, \ldots, S_{n}, Z_{n}\right)$ is a maximal g-layering, then $Z_{0}$ is either shallow relative to $S_{1}$ or acylindrical.

Proof. This is an immediate consequence of Lemmas 5.3 and 5.4.

Theorem 5.1. Suppose $M$ is a closed, simple 3-manifold containing a separating connected closed incompressible surface of some genus $g$, that $\mathrm{Hg}(M) \geq g+4$, and that $M$ is $\left(g, \frac{g}{2}+1\right)$-small. Then $M$ contains a separating connected closed incompressible surface $S$ of genus $g$ satisfying at least one of the following conditions:

(1) at least one component of $M \backslash \backslash S$ is acylindrical; or

(2) for each component $B$ of $M \backslash \backslash S$ we have $\operatorname{kish}(B) \neq \emptyset$.

Proof. It follows from the Haken finiteness theorem [9, Lemma 13.2] that the set of all depths of $g$-layerings in $M$ is bounded. In particular, any $g$-layering has a refinement that is a maximal $g$-layering.

By hypothesis $M$ contains some separating connected closed incompressible surface $T$ of genus $g$. If $X$ and $Y$ denote the closures of the components of $M-T$, then $(X, T, Y)$ is a $g$-layering of depth 1 . In particular $M$ contains a $g$-layering, and hence contains a maximal $g$-layering.

Now suppose that the conclusion of Theorem 5.1 does not hold. Fix a maximal $g$-layering $\left(Z_{0}, S_{1}, Z_{1}, \ldots, S_{n}, Z_{n}\right)$. Then neither $Z_{0}$ nor $Z_{n}$ is acylindrical, since otherwise $S=S_{1}$ or $S_{n}$ would satisfy conclusion (1) of the theorem. In view of Lemmas 5.4 and 5.5, and the hypothesis that $M$ is $\left(g, \frac{g}{2}+1\right)$-small, it follows that $Z_{0}$ and $Z_{n}$ are both shallow.

For $0<i \leq n$ we define $B_{i}^{-}=Z_{0} \cup Z_{1} \cup \cdots \cup Z_{i-1}$ and $B_{i}^{+}=Z_{i} \cup \cdots \cup$ $Z_{n}$. For each $i$, we must have either $\operatorname{kish}\left(B_{i}^{-}\right)=\emptyset$ or $\operatorname{kish}\left(B_{i}^{+}\right)=\emptyset$, since otherwise $S=S_{i}$ would satisfy conclusion (2) of the theorem. Hence by the observation made in 5.1, at least one of $B_{i}^{-}$or $B_{i}^{+}$has the form $|\mathcal{W}|$ for some book of $I$-bundles $\mathcal{W}$. Since $M$ is in particular $\left(g, \frac{g+1}{2}\right)$-small, it now follows from Lemma 5.2 that at least one of $B_{i}^{-}$or $B_{i}^{+}$is shallow. 
Since $B_{0}^{-}=Z_{0}$ is shallow, there is a largest index $k \leq n$ such that $B_{k}^{-}$ is shallow. Since $B_{k}^{-}$is shallow, it follows from $\operatorname{Lemma} 4.3$ that $\operatorname{Hg}\left(B_{k}^{-}\right) \leq$ $g+1$. We distinguish two cases depending on whether $k<n$ or $k=n$.

If $k<n$ then $B_{k+1}^{-}$is not shallow, and hence $B_{k+1}^{+}$is shallow. By the definition of $B_{k+1}^{-}$, the frontier of $Z_{k}$ in $B_{k+1}^{-}$is the closed surface $S_{k}$, and by the definition of a $g$-layering, $Z_{k}$ is shallow relative to $S_{k}$. We may thus apply Lemma 4.2 with $Q=B_{k+1}^{-}$and $Z=Z_{k}$ to deduce that

$$
\operatorname{Hg}\left(B_{k+1}^{-}\right) \leq 1+\operatorname{Hg}\left(B_{k}^{-}\right) \leq g+2
$$

Now since $B_{k+1}^{+}$is shallow, we may again apply Lemma 4.2 , this time with $Q=M$ and $Z=B_{k+1}^{+}$, to deduce that

$$
\operatorname{Hg}(M) \leq 1+\operatorname{Hg}\left(B_{k+1}^{-}\right) \leq g+3
$$

This contradicts the hypothesis.

Now suppose that $k=n$. In this case, since $B_{n}^{+}=Z_{n}$ is shallow, we may apply Lemma 4.2 with $Q=M$ and $Z=Z_{n}$ to deduce that

$$
\operatorname{Hg}(M) \leq 1+\operatorname{Hg}\left(B_{n}^{-}\right) \leq g+2
$$

Once again we have a contradiction to the hypothesis.

Corollary 5.1. Suppose that $M$ is a closed, simple 3-manifold that contains a connected closed incompressible surface of genus 2 , and that $\operatorname{Hg}(M) \geq 6$. Then $M$ contains a connected closed incompressible surface $S$ of genus 2 such that either $M \backslash \backslash S$ has an acylindrical component, or $\bar{\chi}(\operatorname{kish}(M \backslash \backslash S)) \geq 2$.

Proof. Since $M$ is simple, it is $(2,2)$-small.

First consider the case in which $M$ contains a separating, connected, closed, incompressible surface of genus 2 . Since $M$ is $(2,2)$-small, Theorem 5.1 gives a separating connected closed incompressible surface $S$ of genus 2 such that either at least one component of $M \backslash \backslash S$ is acylindrical, or for each component $B$ of $M \backslash \backslash S$ we have $\operatorname{kish}(B) \neq \emptyset$. In particular, $\operatorname{kish}(M \backslash \backslash S)$ has at least two components. By Definition 1.1, each component of $\operatorname{kish}(M \backslash \backslash S)$ has a strictly negative Euler characteristic. Hence $\bar{\chi}(\operatorname{kish}(M \backslash \backslash S)) \geq 2$.

Now suppose that $M$ contains no separating, connected, closed, incompressible surface of genus 2. In this case $M$ is $(3,2)$-small. By hypothesis, 
$M$ contains a connected, closed, incompressible surface $S$ of genus 2 , which must be non-separating. It now follows from Theorem 3.1 that $\bar{\chi}(\operatorname{kish}(M \backslash \backslash S)) \geq 2$.

\section{Volume bounds}

Recall that a slope on a torus $T$ is an unoriented isotopy class of homotopically non-trivial simple closed curves on $T$. If the torus $T$ is a boundary component of an orientable 3-manifold $N$, and $r$ is a slope on $T$, we denote by $N(r)$ the "Dehn-filled" manifold obtained from the disjoint union of $N$ with $D^{2} \times S^{1}$ by gluing $\left(\partial D^{2}\right) \times S^{1}$ to $\partial N$ via a homeomorphism which maps $\left(\partial D^{2}\right) \times\{$ point $\}$ to a curve representing the slope $r$.

Lemma 6.1. Let $N$ be a compact 3-manifold whose boundary is a single torus, let $S \subset N$ be a closed connected incompressible surface, and let $p$ be a prime. Then there exist infinitely many slopes $r$ on $\partial N$ for which the following conditions hold:

(1) the inclusion homomorphism $H_{1}\left(N ; \mathbb{Z}_{p}\right) \rightarrow H_{1}\left(N\left(r_{i}\right) ; \mathbb{Z}_{p}\right)$ is an isomorphism; and

(2) $S$ is incompressible in $N\left(r_{i}\right)$.

Proof. There is a natural bijective correspondence between slopes on $\partial M$ and unordered pairs of the form $\{c,-c\}$ where $c$ is a primitive element of $L \doteq H_{1}(\partial N ; \mathbb{Z})$. If $r$ is a slope, the elements of the corresponding unordered pair are the homology classes defined by the two orientations of a simple closed curve representing $c$. If $c$ is a primitive element of $L$ we shall denote by $r_{c}$ the slope corresponding to the pair $\{c,-c\}$.

Let $K \subset L$ denote the kernel of the natural homomorphism $H_{1}\left(\partial N ; \mathbb{Z}_{p}\right) \rightarrow H_{1}\left(N ; \mathbb{Z}_{p}\right)$. If $c$ is a primitive class $c \in K$, it follows from the Mayer-Vietoris theorem that the inclusion homomorphism $H_{1}\left(N ; \mathbb{Z}_{p}\right) \rightarrow$ $H_{1}\left(N\left(r_{c}\right) ; \mathbb{Z}_{p}\right)$ is an isomorphism.

We fix a basis $\{\lambda, \mu\}$ of $L$ such that $\lambda \in K$, and we identify $L$ with an additive subgroup of the 2-dimensional real vector space $V=H_{1}(\partial N ; \mathbf{R})$. For each positive integer $n$, let $A_{n} \subset V$ denote the affine line $\mathbf{R} \lambda+n p \mu \subset V$. Then $A_{n} \cap L \subset K$, and $A_{n} \cap L$ contains infinitely many primitive elements of $L$ (for example the elements of the form $(k n p+1) \lambda+n p \mu$ for $k \in \mathbb{Z}$ ). In particular, $K$ contains infinitely many primitive elements of $L$.

We distinguish two cases, depending on whether there (a) does or (b) does not exist an annulus in $M$ having one boundary component in $S$ and one 
in $\partial M$, and having interior disjoint from $S \cup \partial M$. In case (a) it follows from [5, Theorem 2.4.3] that there is a slope $r_{0}$ such that for every slope $r$ whose geometric intersection number with $r$ is $>1$, the surface $S$ is incompressible in $M(r)$. (For the application of [5, Theorem 2.4.3] we need to know that $S$ is not boundary parallel in $M$, but this is automatic since $S$ has genus 2.) In particular, there are three affine lines $B_{1}, B_{2}$ and $B_{3}$ in $V$ such that for any primitive class $c \in L \backslash\left(B_{1} \cup B_{2} \cup B_{3}\right)$, the surface $S$ is incompressible in $M\left(r_{c}\right)$. If we choose a natural number $n$ large enough so that $A_{n}$ is distinct from $B_{1}, B_{2}$ and $B_{3}$, then $A_{n} \cap\left(B_{1} \cup B_{2} \cup B_{3}\right)$ consists of at most three points. Hence of the infinitely many primitive elements of $L$ belonging to $A_{n} \cap L$, at most three lie in $B_{1} \cup B_{2} \cup B_{3}$. For any primitive element $c \in\left(A_{n} \cap L\right) \backslash\left(B_{1} \cup B_{2} \cup B_{3}\right)$, the slope $r_{c}$ satisfies conclusions (1) and (2).

In case (b) it follows from [19, Theorem 1] that there are at most three slopes $r$ for which $S$ is compressible in $M(r)$. In particular, of the infinitely many elements $c \in K$ which are primitive in $L$, all but finitely many have the property that $S$ is incompressible in $M\left(r_{c}\right)$. Hence there are infinitely many slopes $c$ satisfying conclusions (1) and (2).

Definition 6.1. If $X$ is a compact orientable manifold with non-empty boundary then by the double of $X$ we shall mean the quotient space $D X$ obtained from $X \times\{0,1\}$ by identifying $(x, 0)$ with $(x, 1)$ for each $x \in \partial X$. The involution of $X \times\{0,1\}$ which interchanges $(x, 0)$ and $(x, 1)$ induces an orientation-reversing involution $\tau: D X \rightarrow D X$, which we shall call the canonical involution of $D X$. Define geodvol $X=\frac{1}{2} v_{3}\|[D X]\|$, where $\|[D X]\|$ denotes the Gromov norm of the fundamental class of $D X$, and $v_{3}$ is the volume of a regular ideal tetrahedron.

The following standard result does not seem to be in the literature.

Proposition 6.1. Let $X$ be a compact connected orientable 3-manifold with connected boundary $S$ of genus greater than 1 . Suppose that $X$ is irreducible, boundary irreducible and acylindrical. Then $X$ admits a hyperbolic metric with totally geodesic boundary, and geodvol $X$ is the volume of this metric.

Proof. The closed manifold $D X$ is simple, and the surface $S$ is incompressible in $D X$. Thus $D X$ admits a complete hyperbolic metric by Thurston's Hyperbolization Theorem for Haken manifolds [15]. Let $\tau: D X \rightarrow D X$ be the canonical involution of $D X$. Fix a basepoint $\star \in S$ and a basepoint $\tilde{\star}$ in the universal cover of $D X$ which maps to $\star$. We identify $D X$ with $\mathbb{H}^{3} / \Gamma$, where $\Gamma$ is a Kleinian group. Using the basepoint $\tilde{\star}$, we identify $\pi_{1}(D X, \star)$ 
with $\Gamma$. Let $p: \mathbb{H}^{3} \rightarrow D X$ be the covering projection, and let $\tilde{\tau}: \mathbb{H}^{3} \rightarrow \mathbb{H}^{3}$ denote the lift of $\tau \circ p$ which fixes $\tilde{\star}$. Let $\widetilde{S}$ be the component of $p^{-1}(S)$ which contains $\tilde{\star}$. The map $\tilde{\tau}$ is then an orientation-reversing involution of $\mathbb{H}^{3}$ which fixes $\widetilde{S}$.

Since $\tau$ is a homotopy equivalence, it follows from the proof of Mostow's rigidity theorem ( [13], cf. [4]) that $\tilde{\tau}$ extends continuously to $S_{\infty}^{2}$, and that there is an isometry $\tau^{\prime}$ of $\mathbb{H}^{3}$ whose extension to $S_{\infty}^{2}$ agrees with that of $\tilde{\tau}$. In particular, $\tau^{\prime}$ is an orientation-reversing isometry of $\mathbb{H}^{3}$ whose restriction to $S_{\infty}^{2}$ normalizes the restriction of $\Gamma$ to $S_{2}^{\infty}$. Thus $\tau^{\prime}$ normalizes $\Gamma$ in the isometry group of $\mathbb{H}^{3}$, and consequently $\tau^{\prime}$ induces an involution of $D X$. The restriction of $\tau^{\prime}$ to $S_{\infty}^{2}$ also commutes with the restriction of each isometry in the image $\Delta$ of the inclusion homomorphism $\pi_{1}(S, \star) \rightarrow \Gamma$. It follows that $\tau^{\prime}$ must be a reflection through a hyperbolic plane $\Pi$, where $\Pi$ contains the axis of each element of $\Delta$. In particular $\Pi$ is invariant under $\Delta$. Moreover, since the image of $\Pi$ in $D X$ is contained in the fixed set of an involution of $D X$, it must be a compact subsurface $F$.

Since $F$ is covered by a hyperbolic plane, it is a totally geodesic surface in $D X$. Let $\Delta^{\prime} \leq \Gamma$ denote the stabilizer of $\Pi$. Then the covering space $\widetilde{D X}=\mathbb{H}^{3} / \Delta^{\prime}$ is homeomorphic to $F \times \mathbf{R}$. Let $\widetilde{F}$ denote the image of $\Pi$ in $\widetilde{D X}$, so that $\widetilde{F}$ is the image of a lift of the inclusion of $F$ into $D X$. Since $\Delta \leq \Delta^{\prime}$, the inclusion of $S$ into $D X$ lifts to an embedding of $S$ in $\widetilde{D X}$. Let $\widetilde{S}$ denote the image of this lift. The surfaces $\widetilde{S}$ and $\widetilde{F}$ are $\pi_{1}$-injective and can be isotoped to a pair of disjoint surfaces which cobound a compact submanifold $W$ of $\widetilde{M}$. According to [9, Theorem 10.5] $W$ is homeomorphic to $\widetilde{S} \times I$. This shows that $\widetilde{S}$ is isotopic to $\widetilde{F}$ in $\widetilde{D X}$, and hence that $S$ is homotopic to $F$ in $D X$. Since $S$ is incompressible in $D X$, it follows from [18, Corollary 5.5] that $S$ is isotopic to the totally geodesic surface $F$.

Since $S$ and $F$ are isotopic, there is an ambient isotopy of $D X$ which carries $X$ onto a submanifold of $D X$ bounded by $F$. Pulling back the hyperbolic metric by the time-1 map of this isotopy endows $X$ with the structure of a complete hyperbolic manifold with totally geodesic boundary. The isometry of $D X$ induced by $\tau^{\prime}$ fixes $F$ and exchanges its complementary components. Hence, with the pulled back metric, $X$ has half the hyperbolic volume of $D X$. Since the hyperbolic volume of $D X$ is equal to $v_{3}\|[D X]\|$ (see $[4$, Theorem C.4.2]), this completes the proof.

The result below follows from a result of Agol-Storm-Thurston [2]

Proposition 6.2. Let $M$ be a closed, orientable hyperbolic 3-manifold containing a closed connected incompressible surface $S$ such that $M \backslash \backslash S$ 
has an acylindrical component $X$. Then

$\operatorname{vol} M \geq \operatorname{geodvol} X$.

Proof. Let $g: S \rightarrow M$ denote the inclusion map. Since $S$ is a two-sided embedded surface, the family of all immersions of $S$ in $M$ which are homotopic to $g$ is non-empty. Since $M$ is $\mathbb{P}^{2}$-irreducible, the main result of [8] asserts that this family contains a least area immersion $f: S \rightarrow M$, which is either an embedding or a 2 -sheeted covering of a non-orientable surface. Moreover, the second case arises only if $S$ bounds a twisted $I$-bundle whose 0 -section is isotopic to $f(S)$. Since $f$ is locally area minimizing, $f(S)$ is a minimal surface.

It follows from [18, Corollary 5.5] that if $f$ is an embedding then $f(S)$ is ambiently isotopic to $S$, and if $f$ is a 2-sheeted covering map then $f(S)$ is isotopic to the 0 -section of the twisted $I$-bundle bounded by $S$. Hence one of the components, say $X$, of $M \backslash \backslash f(S)$ is acylindrical. We may identify $X$ with the path completion of a component $X_{0}$ of $M-f(S)$. Then the natural map $X \rightarrow M$ maps the interior of $X$ homeomorphically onto $X_{0}$ and maps $\partial X$ onto $f(S)$, either by a homeomorphism or by a 2 -sheeted cover. The latter possibility arises exactly when $S$ bounds a twisted $I$-bundle and $f(S)$ is a non-orientable surface. In particular, pulling back the hyperbolic metric on $M$ under the natural map $X \rightarrow M$ gives $X$ the structure of a complete hyperbolic manifold whose boundary is a minimal surface. Theorem 7.2 of [2] states that such a manifold $X$ satisfies vol $X \geq \operatorname{geodvol} X$.

Clearly we have vol $M \geq \operatorname{vol} X$, so the proof is complete.

Theorem 6.1. Let $M$ be a closed, orientable hyperbolic 3-manifold containing a closed connected incompressible surface of genus 2 , and suppose that $\operatorname{Hg}(M) \geq 6$. Then $M$ has volume greater than 6.45 .

Proof. According to Corollary 5.1, $M$ contains a connected closed incompressible surface $S$ of genus 2 such that either $M \backslash \backslash S$ has an acylindrical component, or $\bar{\chi}(\operatorname{kish}(M \backslash \backslash S)) \geq 2$. If $M \backslash \backslash S$ has an acylindrical component $X$, by Proposition $6.1 X$ admits a hyperbolic metric with totally geodesic boundary, and the volume of $X$ in this metric is equal to geodvol $X$. The main result of [12] asserts that this volume is greater than 6.45 ; hence by Proposition 6.2, vol $M$ is also greater than 6.45 . On the other hand, if $\bar{\chi}(\operatorname{kish}(M \backslash \backslash S)) \geq 2$, then Theorem 9.1 of [2] implies that $M$ has volume greater than 7.32 . 
The following lemma is a strict improvement on Proposition 10.1 of [7]. The improvement is made possible by the results of [6].

Lemma 6.2. Let $M$ be a complete, finite-volume, orientable hyperbolic 3manifold having exactly one cusp, such that $\operatorname{dim}_{\mathbb{Z}_{2}} H_{1}\left(M ; \mathbb{Z}_{2}\right) \geq 6$. Then either

(1) $\operatorname{vol} M>5.06$, or

(2) $M$ contains a genus-2 connected incompressible surface.

Proof. This is identical to the proof of [7, Proposition 10.1] except that

- each of the two appearances of the number 7 in the latter proof is replaced by 6 , and

- the reference to the case $g=2$ of [1, Theorem 8.13] is replaced by a reference to the case $g=2$ of [6, Theorem 5.1].

Theorem 6.2. Let $M$ be a complete, finite-volume, orientable hyperbolic 3-manifold having exactly one cusp, and suppose that

$$
\operatorname{dim}_{\mathbb{Z}_{2}} H_{1}\left(M ; \mathbb{Z}_{2}\right) \geq 6
$$

Then $M$ has volume greater than 5.06 .

Proof. For a hyperbolic manifold satisfying the hypotheses of Theorem 6.2, Lemma 6.2 asserts that either $M$ has volume greater than 5.06 or $M$ contains a closed connected incompressible surface of genus 2 . In the latter case, let $N$ denote the compact core of $M$. According to Lemma 6.1 there is an infinite sequence of distinct slopes $\left(r_{i}\right)_{i \geq 1}$ on $\partial N$ such that $S$ is incompressible in each $N\left(r_{i}\right)$, and $\operatorname{dim}_{\mathbb{Z}_{2}} H_{1}\left(N\left(r_{i}\right) ; \mathbb{Z}_{2}\right) \geq 6$ for each $i$. The hyperbolic Dehn surgery theorem ( [17], cf. [14]) asserts that $M_{i} \doteq N\left(r_{i}\right)$ is hyperbolic for all sufficiently large $i$, and hence after passing to a subsequence we may assume that all the $M_{i}$ are hyperbolic. We now invoke Theorem 1A of [14], which implies that vol $M_{i}<\operatorname{vol} M$ for all but finitely many $i$. (The authors of [14] attribute this particular consequence of their main result to Thurston.)

Now since $\operatorname{dim}_{\mathbb{Z}_{2}} H_{1}\left(M_{i} ; \mathbb{Z}_{2}\right) \geq 6$ for each $i$, we have in particular that $\operatorname{Hg}\left(M_{i}\right) \geq 6$ for each $i$. Since each $M_{i}$ contains the genus-2 connected closed incompressible surface $S$, it now follows from Theorem 6.1 that vol $M_{i}>6.45$ for each $i$. Hence vol $M>6.45$. 
Theorem 6.3. Let $M$ be a closed, orientable hyperbolic 3-manifold with

$$
\operatorname{dim}_{\mathbb{Z}_{2}} H_{1}\left(M ; \mathbb{Z}_{2}\right) \geq 4
$$

and suppose that the image of the cup product map $H^{1}\left(M ; \mathbb{Z}_{2}\right) \otimes$ $H^{1}\left(M ; \mathbb{Z}_{2}\right) \rightarrow H^{2}\left(M ; \mathbb{Z}_{2}\right)$ has dimension at most 1 . Then $M$ has volume greater than 3.08 .

Proof. We recall that a group $\Gamma$ is said to be $k$-free for a given positive integer $k$ if every subgroup of $\Gamma$ having rank at most $k$ is free. According to Corollary 9.3 of [1], which was deduced from results in [3], if $M$ is a closed, orientable hyperbolic 3 -manifold such that $\pi_{1}(M)$ is 3-free then vol $M>3.08$.

Now suppose that $M$ satisfies the hypotheses of Theorem 6.3 , but that $\pi_{1}(M)$ is not 3 -free. Fix a base point $P \in M$ and a subgroup $X$ of $\pi_{1}(M, P)$ which has rank at most 3 and is not free. Let $\bar{X}$ denote the image of $X$ under the natural homomorphism $\eta: \pi_{1}(M, P) \rightarrow H_{1}\left(M ; \mathbb{Z}_{2}\right)$. Then the subspace $\bar{X}$ of $H_{1}\left(M ; \mathbb{Z}_{2}\right)$ has dimension at most 3 . Since $\operatorname{dim}_{\mathbb{Z}_{2}} H_{1}\left(M ; \mathbb{Z}_{2}\right) \geq 4$, there is a codimension-1 subspace $V$ of $H_{1}\left(M ; \mathbb{Z}_{2}\right)$ containing $\bar{X}$. Then $Y \doteq \eta^{-1}(V)$ is an index-2 subgroup of $\pi_{1}(M, P)$ containing $X$. Hence $Y$ defines a 2-sheeted based covering space $p:(\widetilde{M}, \widetilde{P}) \rightarrow(M, P)$ such that $p_{\sharp}: \pi_{1}(\widetilde{M}, \widetilde{P}) \rightarrow \pi_{1}(M, P)$ maps some subgroup $\widetilde{X}$ of $\pi_{1}(\widetilde{M}, \widetilde{P})$ isomorphically onto $X$. In particular $\widetilde{X}$ has rank at most 3 and is not free, and so $\pi_{1}(\widetilde{M})$ is not 3 -free.

We now invoke Proposition 3.5 of [7], which asserts that if $M$ is a closed, aspherical 3-manifold, if $r=\operatorname{dim}_{\mathbb{Z}_{2}} H_{1}\left(M ; \mathbb{Z}_{2}\right)$, and if $t$ denotes the dimension of the image of the cup product map $H^{1}\left(M ; \mathbb{Z}_{2}\right) \otimes H^{1}\left(M ; \mathbb{Z}_{2}\right) \rightarrow$ $H^{2}\left(M ; \mathbb{Z}_{2}\right)$, then for any integer $m \geq 0$ and any regular covering $\widetilde{M}$ of $M$ with covering group $\left(\mathbb{Z}_{2}\right)^{m}$, we have $\operatorname{dim}_{\mathbb{Z}_{2}} H_{1}\left(\widetilde{M} ; \mathbb{Z}_{2}\right) \geq(m+1) r-$ $m(m+1) / 2-t$. Taking $M$ and $\widetilde{M}$ as above, the hypotheses of of $[7$, Proposition 3.5] hold with $m=1$, and by the hypothesis of the present theorem we have $r \geq 4$ and $t \leq 1$. Hence $\operatorname{dim}_{\mathbb{Z}_{2}} H_{1}\left(\widetilde{M} ; \mathbb{Z}_{2}\right) \geq 6$.

We next invoke Proposition 7.1 of [6], which implies that if $k \geq 3$ is an integer and if $N$ is a closed simple 3 -manifold such that $\operatorname{dim}_{\mathbb{Z}_{2}} H_{1}\left(N ; \mathbb{Z}_{2}\right) \geq$ $\max (3 k-4,6)$, then either $\pi_{1}(N)$ is $k$-free, or $N$ contains a closed connected incompressible surface of genus at most $k-1$. We may apply this with $N=$ $\widetilde{M}$ and $k=3$, since we have seen that $\operatorname{dim}_{\mathbb{Z}_{2}} H_{1}\left(\widetilde{M} ; \mathbb{Z}_{2}\right) \geq 6$. Since we have also seen that $\pi_{1}(\widetilde{M})$ is not 3 -free, $\widetilde{M}$ must contain a closed connected incompressible surface $S$ of genus at most 2, and in view of simplicity, $S$ must have genus exactly 2 . Again using that $\operatorname{dim}_{\mathbb{Z}_{2}} H_{1}\left(\widetilde{M} ; \mathbb{Z}_{2}\right) \geq 6-$ so that in particular $\operatorname{Hg}(\widetilde{M}) \geq 6$ - we deduce from Theorem 6.1 , with $\widetilde{M}$ 
playing the role of $M$, that vol $\widetilde{M}>6.45$. Hence

$$
\operatorname{vol} M=\frac{1}{2} \operatorname{vol} \widetilde{M}>3.225>3.08
$$

\section{Acknowledgments}

Research of M.C. was partially supported by NSF grants DMS-0204142 and DMS-0504975, J.B. was partially supported by NSF grant DMS-0703749 and P.B.S. was partially supported by NSF grants DMS-0204142 and DMS0504975 .

\section{References}

[1] I. Agol, M. Culler and P.B. Shalen, Singular surfaces, mod 2 homology, and hyperbolic volume, I. Trans. Amer. Math. Soc., to appear, arXiv:math.GT/0506396.

[2] I. Agol, P.A. Storm and W.P. Thurston, Lower bounds on volumes of hyperbolic Haken 3-manifolds, J. Amer. Math. Soc. 20(4) (2007), 10531077 (electronic), with an appendix by Nathan Dunfield.

[3] J.W. Anderson, R.D. Canary, M. Culler and P.B. Shalen, Free Kleinian groups and volumes of hyperbolic 3-manifolds, J. Differential Geom. 43(4) (1996), 738-782.

[4] R. Benedetti and C. Petronio, Lectures on hyperbolic geometry, Universitext, Springer-Verlag, Berlin, 1992.

[5] M. Culler, C.McA. Gordon, J. Luecke and P.B. Shalen, Dehn surgery on knots, Ann. Math. (2), 125(2) (1987), 237-300.

[6] M. Culler and P.B. Shalen, Singular surfaces, mod 2 homology, and hyperbolic volume, II, Preprint, arXiv:math/0701666v5, May 2008.

[7] M. Culler and P.B. Shalen, Volume and homology of one-cusped hyperbolic 3-manifolds, Algebr. Geom. Topol. 8(1) (2008), 343-379.

[8] M. Freedman, J. Hass and P. Scott, Least area incompressible surfaces in 3-manifolds, Invent. Math. 71(3) (1983), 609-642.

[9] J. Hempel, 3-manifolds, AMS Chelsea Publishing, Providence, RI, 2004. Reprint of the 1976 original. 
[10] W.H. Jaco and P.B. Shalen, Seifert fibered spaces in 3-manifolds, Mem. Amer. Math. Soc. 21(220) (1979), viii+192.

[11] K. Johannson, Homotopy equivalences of 3-manifolds with boundaries, Lecture Notes in Mathematics, 761, Springer, Berlin, 1979.

[12] S. Kojima and Y. Miyamoto, The smallest hyperbolic 3-manifolds with totally geodesic boundary, J. Differential Geom. 34(1) (1991), 175-192.

[13] G.D. Mostow, Quasi-conformal mappings in $n$-space and the rigidity of hyperbolic space forms, Inst. Hautes Études Sci. Publ. Math. 34 (1968), 53-104, MR 0236383 (38 \#4679).

[14] W.D. Neumann and D. Zagier, Volumes of hyperbolic three-manifolds, Topology 24(3) (1985), 307-332.

[15] J.P. Otal The hyperbolization theorem for fibered 3-manifolds, SMF/AMS Texts and Monographs, 7, American Mathematical Society, Providence, RI, 2001. Translated from the 1996 French original by Leslie D. Kay.

[16] M. Scharlemann and A. Thompson, Heegaard splittings of (sur face) $\times I$ are standard, Math. Ann. 295(3) (1993), 549-564.

[17] W.P. Thurston, The geometry and topology of 3-manifolds, Mimeographed Lecture notes, 1979.

[18] F. Waldhausen, On irreducible 3-manifolds which are sufficiently large, Ann. of Math. (2) 87 (1968), 56-88.

[19] Y.Q. Wu, Incompressibility of surfaces in surgered 3-manifolds, Topology 31(2) (1992), 271-279.

Department of Mathematics, Statistics, and Computer Science

(M/C 249)

UNIVERSITY of Illinois AT CHICAGO

851 S. Morgan St.

ChicAgo, IL 60607-7045

USA

E-mail address: culler@math.uic.edu; jdeblois@math.uic.edu;

shalen@math . uic.edu

Received August 3, 2008 